\title{
Structural dynamics of membrane proteins
}

\author{
${\text { So } \text { Iwata }^{1,2}}^{1,2}$
}

${ }^{1}$ Department Of Cell Biology Graduate School Of Medicine, Kyoto University, Kyoto, Japan, ${ }^{2}$ RIKEN SPring-8 Center, Hyogo, Japan E-mail: s.iwata@mfour.med.kyoto-u.ac.jp

The results of genome sequencing projects have shown that up to $30 \%$ of human proteins occur in cell membranes. Membrane proteins play crucial roles in many biological functions, including the capture of energy from sunlight by plants, the use of energy in cells, and the movement of molecules across cell membranes. It is essential to study their four dimensional structures, including dynamics to elucidate the function of these molecules.

At the Japanese XFEL facility, SACLA, we are currently developing a data collection system focusing on dynamic crystallography putting a particular effort on membrane proteins. An experimental system for pump-probe experiments based on serial femtosecond crystallography using a viscous material injector has been developed[1]. After a success to visualize a protein motion in bacteriorhodopsin[2], we have used the system to study the light-induced structural changes and the site of $\mathrm{O}=\mathrm{O}$ bond formation in PSII[3].

Photosystem II (PSII) is a huge membrane-protein complex consisting of 20 different subunits with a total molecular mass of $350 \mathrm{kDa}$ for a monomer. It catalyses light-driven water oxidation at its catalytic centre, the oxygen-evolving complex, OEC. Previously, the structure of PSII has been analysed at $1.9 \AA$ resolution by synchrotron radiation $X$-rays, which revealed that the $\mathrm{OEC}$ is a $\mathrm{Mn} 4 \mathrm{CaO} 5$ cluster organized in an asymmetric, 'distorted-chair' form. The mechanism of $\mathrm{O}=\mathrm{O}$ bond formation, however, remains obscure owing to the lack of intermediate-state structures. We studied the structural changes in PSII induced by two-flash illumination at room temperature at a resolution of $2.35 \AA$ using time-resolved serial femtosecond crystallography. A difference Fourier map between the two-flash and dark-adapted states revealed two areas of apparent changes: around the QB/non-haem iron and the Mn4CaO5 cluster. The changes around the QB/non-haem iron region reflected the electron and proton transfers induced by the two-flash illumination. In the region around the OEC, a water molecule located $3.5 \AA$ from the $\mathrm{Mn} 4 \mathrm{CaO} 5$ cluster disappeared from the map after two-flash illumination. This reduced the distance between another water molecule and the oxygen atom 04 , suggesting that proton transfer also occurred. Importantly, the two-flash-minus-dark difference Fourier map showed an apparent positive peak around 05, a unique $\mu 4-$ oxo-bridge between $\mathrm{Mn} 1$ and Mn4 ions. This indicates the insertion of a new oxygen atom (O6) close to O5, providing an $\mathrm{O}=\mathrm{O}$ distance of $1.5 \AA$ between these two atoms. This mechanism for the $\mathrm{O}=\mathrm{O}$ bond formation is consistent with that proposed previously.

[1]Tono K. et al. (2015) J Synchrotron Radiat.1;22(Pt3),532-537

[2]Nango E. et al. (2016) Science 354(6319);1552-1557

[3]Suga M. et al. (2017) Nature Published online

Keywords: XFEL, Photosystem II, serial femtosecond crystallography 